\title{
USP51 deubiquitylates H2AK13,15ub and regulates DNA damage response
}

\author{
Zhiquan Wang, ${ }^{1,5}$ Honglian Zhang, ${ }^{1,5}$ Ji Liu, ${ }^{1}$ Abigael Cheruiyot, ${ }^{2}$ Jeong-Heon Lee, ${ }^{1,3}$ Tamas Ordog, ${ }^{3}$ \\ Zhenkun Lou, ${ }^{4}$ Zhongsheng You, ${ }^{2}$ and Zhiguo Zhang ${ }^{1,3}$ \\ ${ }^{1}$ Department of Biochemistry and Molecular Biology, Mayo Clinic College of Medicine, Rochester, Minnesota 55905, USA; \\ ${ }^{2}$ Department of Cell Biology and Physiology, Washington University School of Medicine, St. Louis, Missouri 63110, USA; ${ }^{3}$ Center \\ for Individualized Medicine, Mayo Clinic College of Medicine, Rochester, Minnesota 55905, USA; ${ }^{4}$ Division of Oncology Research, \\ Mayo Clinic College of Medicine, Rochester, Minnesota 55905, USA
}

Dynamic regulation of RNF168-mediated ubiquitylation of histone H2A Lys13,15 (H2AK13,15ub) at DNA doublestrand breaks (DSBs) is crucial for preventing aberrant DNA repair and maintaining genome stability. However, it remains unclear which deubiquitylating enzyme (DUB) removes H2AK13,15ub. Here we show that USP51, a previously uncharacterized DUB, deubiquitylates H2AK13,15ub and regulates DNA damage response. USP51 depletion results in increased spontaneous DNA damage foci and elevated levels of H2AK15ub and impairs DNA damage response. USP51 overexpression suppresses the formation of ionizing radiation-induced 53BP1 and BRCA1 but not RNF168 foci, suggesting that USP51 functions downstream from RNF168 in DNA damage response. In vitro, USP51 binds to H2A-H2B directly and deubiquitylates $\mathrm{H} 2 \mathrm{AK13}, 15 \mathrm{ub}$. In cells, USP51 is recruited to chromatin after DNA damage and regulates the dynamic assembly/disassembly of 53BP1 and BRCA1 foci. These results show that USP51 is the DUB for $\mathrm{H} 2 \mathrm{AK} 13,15 \mathrm{ub}$ and regulates DNA damage response.

[Keywords: DNA damage response; H2AK15ub; USP51; deubiqutylating enzyme]

Supplemental material is available for this article.

Received September 13, 2015; revised version accepted March 21, 2016.

To meet constant insults on the genome from DNA-damaging agents and DNA replication stress, cells have evolved a sophisticated pathway called DNA damage response (DDR) to detect, signal, and repair DNA break lesions (Branzei and Foiani 2008; Huen and Chen 2010; Jackson and Durocher 2013; Marechal and Zou 2013). Defects in DDR have been implicated in a broad spectrum of human diseases, including cancer, infertility, immunological defects, and aging (Jackson and Bartek 2009; Ghosal and Chen 2013). Therefore, it is very important to understand how DDR pathways are regulated. Earlier studies on DDR have documented a kinase cascade to relay DNA damage signaling. For instance, in response to DNA double-strand breaks (DSBs), the most lethal damage that cells encounter, checkpoint kinases ATM/ATR are activated to phosphorylate histone $\mathrm{H} 2 \mathrm{~A}$ variant $\mathrm{H} 2 \mathrm{AX}$ as well as other proteins, including DNA damage mediator MDC1 and two downstream kinases, Chk1 and Chk2 (Ciccia and Elledge 2010). In addition to the kinase cascade, a ubiquitin-dependent signaling cascade has emerged as an important regulator of DDR through its actions on chromatin, the physiological substrates of DNA repair pathways.

\footnotetext{
${ }^{5}$ These authors contributed equally to this work. Corresponding author: zhang.zhiguo@mayo.edu Article is online at http://www.genesdev.org/cgi/doi/10.1101/gad.271841. 115 .
}

The initial event in the ubiquitylation cascade is the recruitment of E3 ligase RNF8 by phosphorylated MDC1 (Huen et al. 2007; Kolas et al. 2007; Mailand et al. 2007; Wang and Elledge 2007). RNF8 ubiquitylates histones $\mathrm{H} 1$ (Thorslund et al. 2015), and ubiquitylated histone $\mathrm{H1}$ products help recruit RNF168, another E3 ubiquitin ligase that is mutated in RIDDLE syndrome, to the chromatin surrounding DNA damage sites (Doil et al. 2009; Stewart et al. 2009). The primary target of RNF168 is Lys13 and Lys 15 of histone H2A (H2AK13,15) (Gatti et al. 2012; Mattiroli et al. 2012). The RNF8- and RNF168-mediated ubiquitylation cascade facilitates the recruitment of DNA repair proteins such as $53 \mathrm{BP} 1$ and BRCA1. 53BP1 is recruited to DNA damage sites by recognizing ubiquitylated H2AK15 (H2AK15ub)-containing nucleosomes through its ubiquitylation-dependent recruitment motif (Fradet-Turcotte et al. 2013) and dimethylated H4K20 (H4K20me2) through its tandem Tudor domains (Botuyan et al. 2006). Currently, it is still unclear how RNF168-mediated $\mathrm{H} 2 \mathrm{AK} 13,15 \mathrm{ub}$ regulates the recruitment of BRCA1 to DNA damage sites. Once recruited, 53BP1 and BRCA1

(C) 2016 Wang et al. This article is distributed exclusively by Cold Spring Harbor Laboratory Press for the first six months after the full-issue publication date (see http://genesdev.cshlp.org/site/misc/terms.xhtml). After six months, it is available under a Creative Commons License (Attribution-NonCommercial 4.0 International), as described at http:// creativecommons.org/licenses/by-nc/4.0/. 
play key roles in DDR through the nonhomologous endjoining (NHEJ) and homologous recombination (HR) pathways, respectively (Bunting et al. 2010). Therefore, the RNF8- and RNF168-dependent ubiquitylation cascade is important for both NHEJ and HR.

Recent studies have shown that the degree of ubiquitylation at DNA damage sites is regulated at multiple levels in order to generate proper DDR. Uncontrolled histone ubiquitylation may result in unscheduled transcriptional silencing, defects in DNA damage checkpoint activation and cell cycle arrest, and genomic instability (Jackson and Durocher 2013). For instance, the RNF168 levels are tightly controlled by E3 ligases TRIP12 and UBR5 to prevent excessive ubiquitylation (Gudjonsson et al. 2012), which can lead to hyperaccumulation of 53BP1 and BRCA1 at DNA damage sites. The deubiquitylating enzyme (DUB) OTUB1 binds UBC13, the ubiquitin-conjugating enzyme for RNF168, and inhibits polyubiquitylation independently of its catalytic activity (Nakada et al. 2010). In addition, several other DUBs have been shown to be involved in DDR. For instance, USP3 and USP44 have been suggested to counteract DSB-induced histone ubiquitylation (Nicassio et al. 2007; Doil et al. 2009; Mosbech et al. 2013; Lancini et al. 2014; Sharma et al. 2014). Overexpression of USP3 and USP44 affects the localization of RNF168 at DSB sites, suggesting that these two DUBs regulate ubiquitylation upstream of RNF168. Similarly, USP16 is proposed to have a role in the removal of H2AK119ub, a histone mark associated with transcriptional silencing (Joo et al. 2007; Shanbhag et al. 2010). These results indicate that chromatin ubiquitylation is regulated at multiple levels by multiple mechanisms, highlighting the importance and complexity of the regulation of chromatin ubiquitylation during DDR. Despite these advances, it is largely unknown which DUB is directly involved in the deubiquitylation of histone H2AK15ub, a critical histone modification involved in DDR.

Here, we report that USP51, a previously uncharacterized protein, is involved in DDR by removing H2AK13,15ub following DNA repair. Depletion of USP51 results in increased H2AK13,15ub levels on chromatin and delayed disassembly of proteins at DNA damage foci. Together, these studies identify a novel, bona fide DUB that regulates genome integrity by modulating H2AK13,15ub levels.

\section{Results}

\section{Depletion of USP51 results in increased DNA} damage foci

In a shRNA screen for the regulators of nucleosome assembly (H Zhang, H Gan, Z Wang, J Lee, T Ordog, M Wold, M Ljungman, and Z Zhang, in prep.), we unexpectedly observed that cells depleted of USP51, a previously uncharacterized DUB, exhibited an increase in spontaneous DNA damage foci (Fig. 1). The DDR proteins 53BP1 and BRCA1 form foci at DNA damage sites, which colocalize with ubiquitin Lys63-conjugated polyubiquitin (K63ub) foci and depend on the RNF8/RNF168-catalyzed ubiquitin pathway (Huen and Chen 2010; Jackson and Durocher 2013; Panier and Boulton 2014). As shown in Figure 1, A and B, depletion of USP51 using two independent shRNAs resulted in a marked increase in 53BP1, BRCA1, and K63ub foci in U2OS cells while having no apparent effect on the protein levels of 53BP1 and BRCA1 (Supplemental Fig. S1A). The 53BP1 foci colocalized with K63ub foci, supporting the idea that depletion of USP51 resulted in the accumulation of spontaneously damaged foci. Moreover, the increased 53BP1 and BRCA1 foci were abolished in RNF168-depleted cells (Supplemental Fig. S1B-D). Importantly, expression of shRNA-resistant USP51, but not a catalytically inactive mutant (USP51/CI), suppressed the 53BP1 foci induced by USP51 depletion (Fig. 1C,D). We also observed that USP51-depleted cells exhibited an increase in G1-phase cells and a reduction in S-phase cells (Supplemental Fig. S1E,F). These results indicate that USP51 and its deubiquitylating enzymatic activity are important for maintaining genome integrity and cell cycle progression.

USP51 functions downstream from MDC1 recruitment in response to ionizing radiation (IR)-induced DNA breaks

Dynamic regulation of protein ubiquitylation at DNA damage foci is important for DDR (Jackson and Durocher 2013). To determine whether USP51 has any direct role in this process, we first tested whether overexpression of USP51 affects IR-induced DNA damage foci. In response to IR, H2AX is phosphorylated at Ser139 $(\gamma \mathrm{H} 2 \mathrm{AX})$ at chromosome breaks, which initiates a protein assembly cascade, resulting in sequential recruitments of MDC1, E3 ubiquitin ligases RNF8 and RNF168, and repair proteins BRCA1 and 53BP1 to DNA damage sites (Huen and Chen 2010). Overexpression of USP51 but not USP51/CI suppressed IR-induced DNA damage foci, including 53BP1 and BRCA1 foci as well as ubiquitin foci detected by FK2 antibodies (Fig. 2A,B). These IR-induced foci colocalized with $\gamma \mathrm{H} 2 \mathrm{AX}$ foci and were dependent on the presence of RNF168 (Supplemental Fig. S2A). In contrast, USP51 overexpression had no apparent effect on the formation of IR-induced MDC1 and $\gamma \mathrm{H} 2 \mathrm{AX}$ foci (Fig. 2C,D; Supplemental Fig. S2B,C). These results suggest that USP51 functions downstream from H2AX phosphorylation and MDC1 recruitment, possibly deubiquitylating a factor during DDR. Consistent with this interpretation, overexpression of USP51, but not its catalytically inactive mutant, also suppressed IR-induced RNF169 foci, which are recruited to DNA damage sites downstream from RNF168 (Supplemental Fig. S2D-F; Shao et al. 2009; Panier et al. 2012; Poulsen et al. 2012).

USP3, USP16, and USP51 function distinctly in response to IR-induced DNA breaks

Previous studies have shown that two other DUBs, USP3 and USP16, are also involved in DDR (Nicassio et al. 2007; Lancini et al. 2014; Sharma et al. 2014). It is known that USP16 targets H2AK119ub and H2AK15ub and that USP3 targets H2AK15ub (Sharma et al. 2014; Zhang 
Wang et al.

A
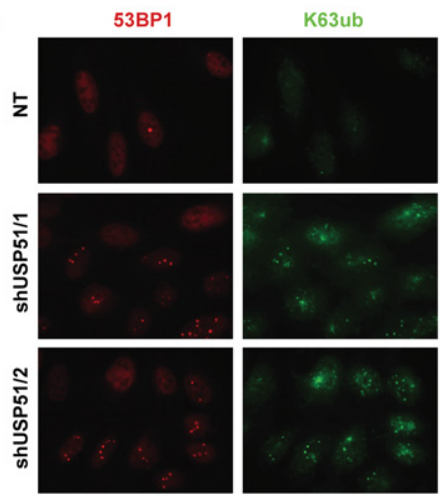

B

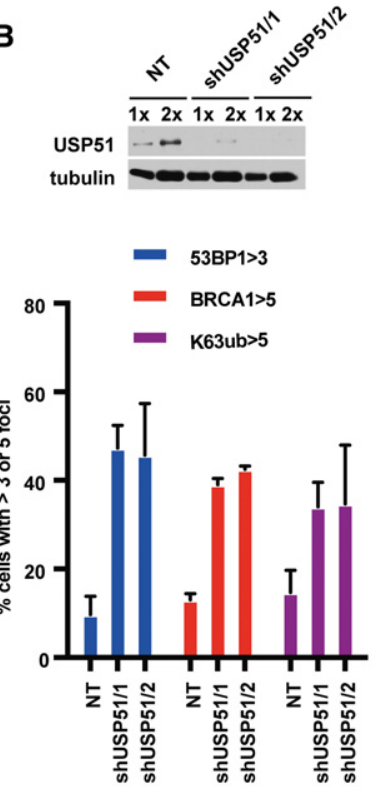

53BP1/K63ub/DAPI
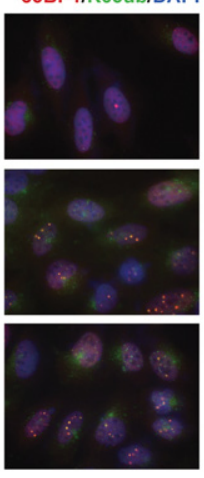

C
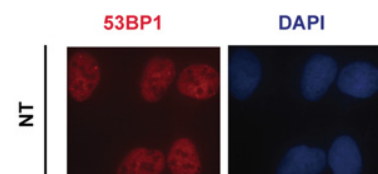

BRCA1

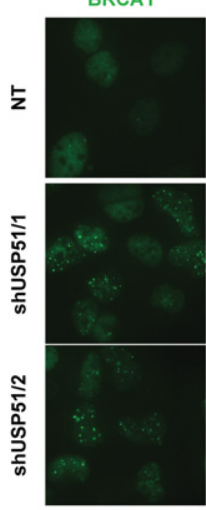

D
DAPI

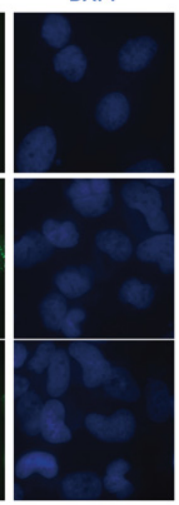

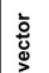

tubulin - - -

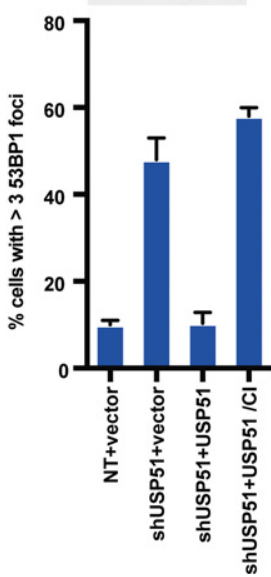

Figure 1. USP51 depletion results in the formation of spontaneous DDR foci. $(A, B)$ Depletion of USP51 results in the formation of DDR foci, including 53BP1, K63ub, and BRCA1 foci. U2OS cells infected with two different shRNAs (shUSP51/1 and shUSP51/2) or nontarget shRNA (NT) were stained with antibodies against 53BP1, K63ub, and BRCA1. Representative images are shown in $A$. The percentage of cells showing more than three (53BP1) or five (BRAC1 and K63ub) foci from three independent experiments is shown in the bottom panel of $B$. USP51 knockdown efficiency is shown in the top panel of $B .(C, D)$ UPS51 catalytic activity is necessary to prevent the formation of 53BP1 foci in response to USP51 depletion. The shRNA-resistant form of USP51 or catalytically inactive USP51 (USP51/CI) was expressed in USP51-depleted U2OS cells, and immunofluorescence was performed using antibodies against 53BP1. Representative images are shown in $C$. (D) USP51 protein expression and quantification of 53BP1 foci from three independent experiments are shown in the top and bottom panels, respectively. Over 100 cells were counted for each experiment to obtain the results in $B$ and $D$.

et al. 2014). To gain insight into how USP51 functions in DDR, we compared the effect of overexpression of USP3, USP16, and USP51 on the formation of IR-induced 53BP1 and RNF168 foci. Consistent with the previous results (Mosbech et al. 2013), overexpression of USP3, but not USP16, suppressed IR-induced 53BP1 foci (Fig. 2E,F). Interestingly, USP51 had a larger suppressive effect than USP3. In addition, overexpression of USP3 suppressed the recruitment of RNF168 to DNA damage foci (Doil et al. 2009; Mattiroli et al. 2012), whereas overexpression of USP16 had only a minor effect. Importantly, overexpression of USP51 had no apparent effect on the formation of IR-induced RNF168 foci (Fig. 2G,H). The differential effect of these three DUBs on the formation of 53BP1 and
RNF168 foci did not appear to be related to their level of expression (Fig. 2F,H). Taken together, these results suggest that USP16, USP3, and USP51 are involved in the different steps of DDR and USP51 functions downstream from the recruitment of RNF168 to DNA breaks.

\section{USP51 regulates $H 2 A K 13,15 u b$}

The RNF168 E3 ligase ubiquitylates H2A at K13,K15, which in turn triggers the recruitment of DDR proteins such as 53BP1 and BRCA1 to DNA breaks (Doil et al. 2009; Stewart et al. 2009; Gatti et al. 2012; Mattiroli et al. 2012). Therefore, we hypothesized that USP51 may be a specific DUB for H2AK15ub. To test this hypothesis, 

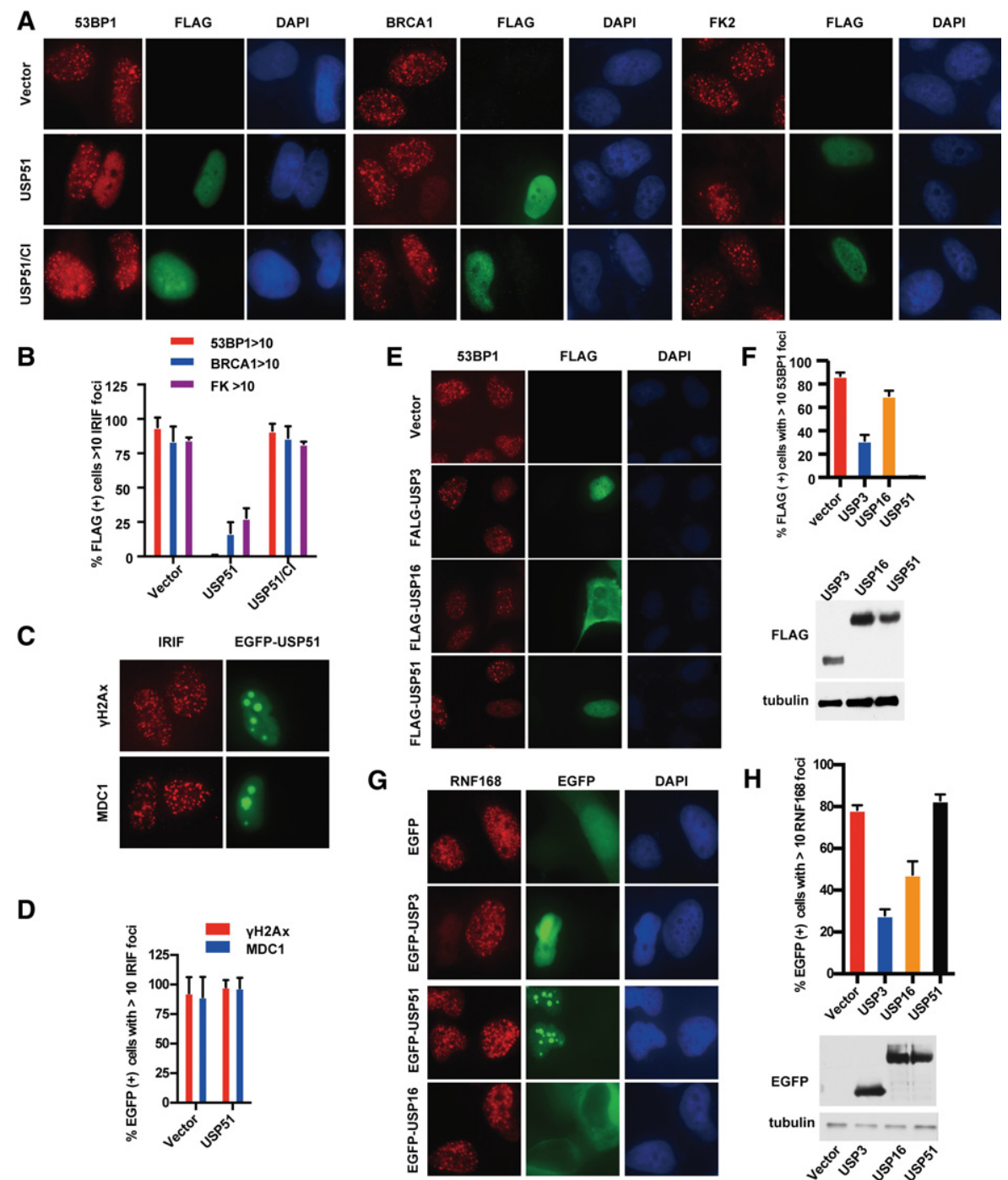

F $\overline{\bar{o}}$
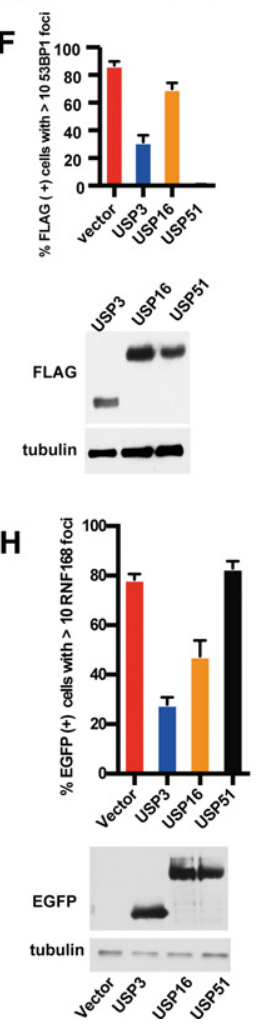

Figure 2. USP51 functions downstream from RNF168 recruitment in response to IR. $(A, B)$ Overexpression of USP51, but not its catalytically inactive mutant, suppresses IR-induced 53BP1, BRCA1, and ubiquitin FK2 foci. U2OS cells were transiently transfected with FlagUSP51 or USP51/CI mutants and exposed to 10 Gy of IR. Immunofluorescence using antibodies against the Flag epitope, 53BP1, BRCA1, and FK2 antibody was performed. Representative images $(A)$ and quantification $(B)$ of IR-induced foci (IRIF) from three independent experiments are shown. $(C, D)$ Overexpression of USP51 fails to suppress IR-induced $\gamma \mathrm{H} 2 \mathrm{AX}$ and MDC1 foci. The experiments were performed as described above except that EGFP-USP51 was used. Note that, unlike Flag-USP51, EGFP-USP51 was also detected in nuclear speckles. $(E, F)$ Overexpression of USP3 and USP51, but not USP16, suppresses IR-induced 53BP1 foci. The experiments were performed as described in $A$, and representative images $(E)$ and quantification $(F)$ of IR-induced 53BP1 foci are shown. $(F$, bottom panel) The expression levels of Flag-USP3, Flag-USP16, and Flag-USP51 were detected by Western blot in U2OS cells. $(G, H)$ Overexpression of USP51 does not suppress IR-induced RNF168 foci. U2OS cells expressing Flag-RNF168 were transiently transfected with EGFP-USP3, EGFPUSP16, or EGFP-USP51 and irradiated at $10 \mathrm{~Gy}$. Immunofluorescence was performed using antibodies against Flag. Representative images $(G)$ and percentage $(H)$ of cells with RNF168 foci are shown. About 50-70 Flag-positive or EGFP-positive cells in each experiment were counted for $B, D, F$, and $H$.

we first immunoprecipitated Flag-H2A under denaturing conditions from cell lines stably expressing Flag-H2A after depletion of USP51 and analyzed ubiquitylated H2A species by Western blotting. USP51 depletion resulted in a marked increase in ubiquitylated H2A (Fig. 3A). Next, we used a cell line expressing Flag-tagged H2A (K118,119R) or H2A (K13,15R) mutants to define which ubiquitylated species are altered by USP51 depletion because H2AK118,119ub and H2AK13,15ub are two ma- jor monoubiquitylated species, with H2AK118,119ub being the dominant one. Briefly, mononucleosomes were immunoprecipitated from these cells using Flag-H2A (K118,119R) or Flag-H2A (K13,15R) mutant proteins, and ubiquitylated species were analyzed by Western blot using antibodies against the Flag epitope. Ubiquitylated H2A from H2A (K118,119R) nucleosomes (Fig. 3B), but not from H2A (K13,15R) nucleosomes (Supplemental Fig. S3A), was elevated upon USP51 depletion. These 
Wang et al.
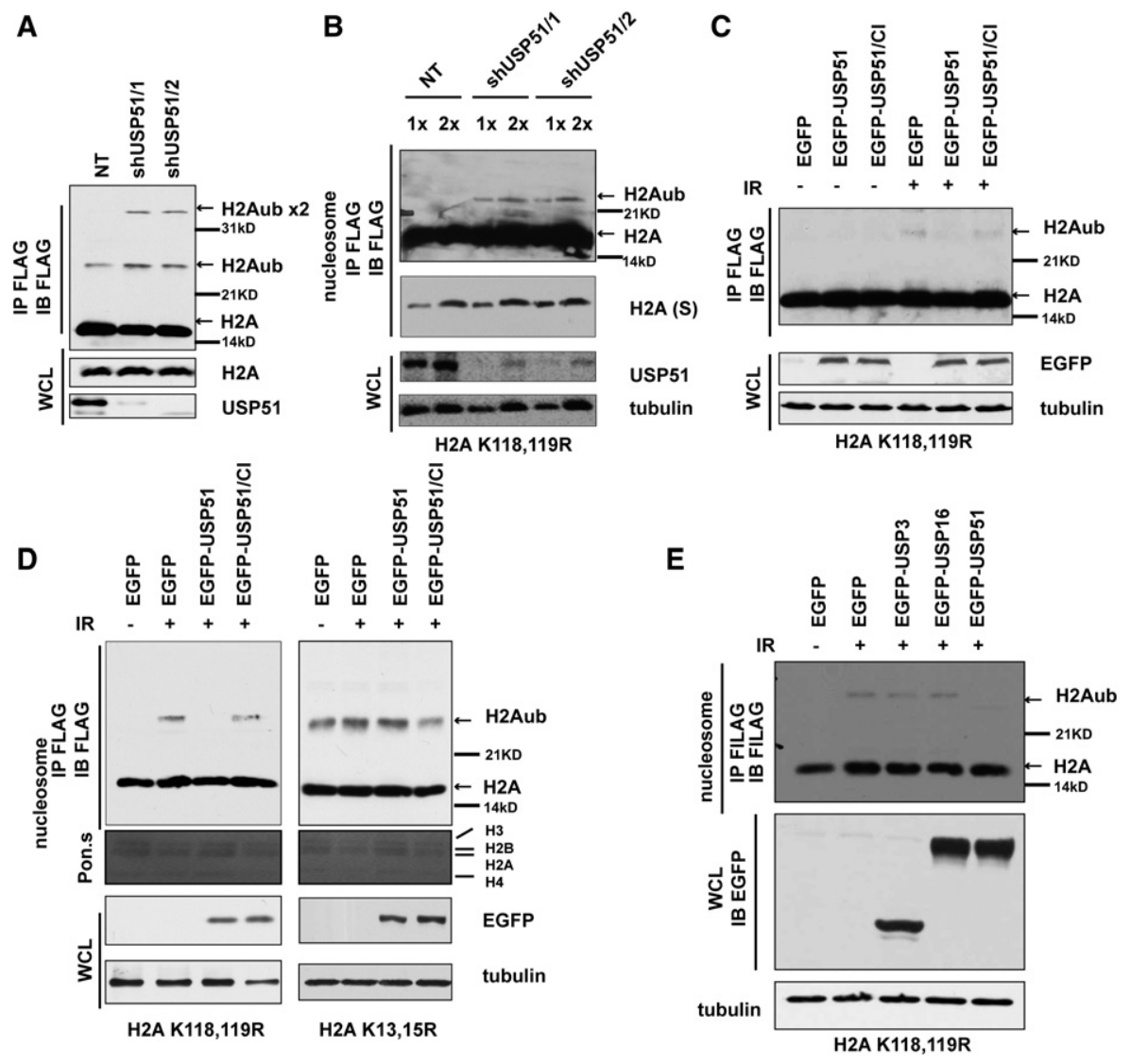

Figure 3. USP51 regulates H2AK13,15ub in 293T cells. $(A, B)$ USP51 depletion results in an increase in H2A ubiquitylation. 293T cell lines stably expressing Flag-tagged H2A or H2A (K118,119R) mutant were infected with lentivirus encoding the indicated shRNAs. $(A)$ After 72 h, Flag-H2A or the mutant was purified under denaturing conditions and analyzed by Western blotting. $(B)$ Mononucleosomes containing Flag-H2A (K118,119R) were purified from the cells expressing Flag-H2A (K118,119R) and analyzed by Western blotting. (WCL) Whole-cell lysate; (arrows) monoubiquitylated and diubiquitylated H2A. $(C, D)$ USP51 overexpression suppresses IR-induced H2AK13,15ub. The Flag-H2A (K118,119R) or Flag-H2A (K13,15R) stable line was transfected with the EGFP-USP51 or EGFP-USP51/ CI mutant. Flag-H2A (under denatured conditions) $(C)$ or mononucleosomes containing the Flag-H2A mutant $(D)$ were purified. Purified proteins were detected by Western blot. (E) Overexpression of USP3 and USP51, but not USP16, suppresses IR-induced H2AK15ub. EGFPUSP3, EGFP-USP16, or EGFP-USP51 was expressed in the Flag-H2A (K118,119R) stable line, and Flag-H2A-containing mononucleosomes were immunoprecipitated. Antibodies used for immunoprecipitation (IP) and immunoblotting (IB) are indicated.

results suggest that USP51 regulates the ubiquitylation of H2AK13,15 but not H2AK118,119.

To provide further evidence supporting the hypothesis that USP51 targets H2AK13,15ub, we asked whether overexpression of USP51 affects H2AK13,15ub levels upon IR, which induces H2AK13,15ub. USP51 was overexpressed in 293T cells stably expressing Flag-H2A (K13,15R) or Flag-H2A (K118,119R). Flag-H2A immunoprecipitation was performed under denaturing conditions (Fig. 3C), or Flag-H2A (K118,119R)-containing or FlagH2A (K13,15R)-containing mononucleosomes were purified (Fig. 3D). Consistent with previous results (Mattiroli et al. 2012), cells treated with IR showed an increase in H2AK13,15ub (Fig. 3C,D), and overexpression of USP51, but not USP51/CI, suppressed IR-induced H2AK13,15ub levels on $\mathrm{H} 2 \mathrm{~A}$ purified under denaturing conditions (Fig. 3C) and H2A in purified mononucleosomes (Fig. 3D, left panel). However, overexpression of USP51 had no apparent effect on the levels of H2AK118,119ub (Fig. 3D, right panel). Thus, USP51 is involved in regulation of IR-induced H2AK13,15ub.

Next, we compared the effect of USP3, USP16, and USP51 overexpression on H2AK13,15ub levels following IR in cells expressing Flag-H2A (K118,119R). Overexpression of USP51 led to a more pronounced reduction in IRinduced H2AK13,15ub than USP3 overexpression (Fig. 3E; Supplemental Fig. S3B), whereas USP16 overexpression had no apparent effects. These results indicate that USP3 and USP51 regulate H2AK13,15ub in response to DNA damage.

\section{USP51 regulates H2AK15ub at DNA damage sites}

While it is known that RNF168 ubiquitylates H2AK13,15, it is not known whether H2AK13,15ub is localized to DNA damage sites in vivo. We generated an H2AK15ubspecific mouse monoclonal antibody using an H2A peptide conjugated at K15 with a ubiquitin peptide 
(Ac-ARAKAK[GGRL]TRSSC). The antibody specifically recognized monoubiquitylated and diubiquitylated $\mathrm{H} 2 \mathrm{~A}$ generated by recombinant RNF168 but not unmodified H2A in vitro (Supplemental Fig. S4A). Moreover, this antibody did not recognize H2AK119ub (Supplemental Fig. S4B) and diubiquitin (Supplemental Fig. S4C). Depletion of USP51, but not USP3 and USP16 in 293T cells (Supplemental Fig. S4D,F) or knockout of USP51 in mouse embryonic stem (ES) cells (Supplemental Fig. S4G), resulted in an increase in ubiquitylated species detected by H2AK15ub antibodies in a RNF168-dependent manner. Similarly, the antibody recognized IR-induced monoubiquitylated and diubiquitylated $\mathrm{H} 2 \mathrm{~A}$ but not $\mathrm{H} 2 \mathrm{~A}$ isolated from 293T cells (Supplemental Fig. S4H). These results are identical to those obtained using H2A mutant lines to detect H2AK15ub (Fig. 3). Together, these studies indicate that the H2AK15ub antibody can recognize ubiquitylated species of H2A catalyzed by RNF168 specifically.

Next, we asked whether H2AK15ub is localized at DNA damage sites using immunofluorescence. In the absence of IR treatment, several foci were revealed using H2AK15ub antibodies. Most of these foci did not appear to have 53BP1 staining. In IR-treated cells, H2AK15ub foci increased dramatically compared with untreated cells. Importantly, the majority of these foci colocalized with 53BP1 foci (Fig. 4A), and depletion of RNF168 resulted in the loss of almost all IR-induced H2AK15ub and 53BP1 foci (Fig. 4A,B). These results provide additional evidence supporting the idea that H2AK15ub antibodies are specific for the recognition of IR-induced H2AK15ub in cells and indicate that H2AK15ub generated by RNF168 is localized at DNA damage sites.

Overexpression of USP51 and USP3, but not USP51/CI or USP16, led to a reduction of H2AK15ub foci and a decrease of total H2AK15ub levels following IR (Fig. 4C, D; Supplemental Fig. S4I). H2AK15ub was also detected at laser-induced DNA damage strips (Fig. 4E,F). Depletion of USP51 resulted in a marked increase in H2AK15ub at laser-induced DNA breaks (Fig. 4E,F). Taken together, these results show for the first time that $\mathrm{H} 2 \mathrm{AK} 15 \mathrm{ub}$ catalyzed by RNF168 is localized at DNA damage sites and indicate that USP51 is the DUB regulating H2AK15ub levels.

\section{USP51 binds to H2A and deubiquitylates H2AK13,15ub in vitro}

To test whether USP51 is an H2AK13,15ub DUB, we first tested whether USP51 can bind to histone H2A. Purified His-tagged USP51 was used to pull down recombinant $\mathrm{H} 2 \mathrm{~A}-\mathrm{H} 2 \mathrm{~B}$ dimers in vitro. His-USP51 bound to H2A, but His-tagged Asf1, a histone H3-H4 chaperone, did not (Fig. 5A), suggesting that USP51 bound to H2A in vitro. In cells, $\mathrm{H} 2 \mathrm{~A}$ and its modified species could be coimmunoprecipitated with EGFP-USP51 (Fig. 5B). These results show that USP51 binds to $\mathrm{H} 2 \mathrm{~A}-\mathrm{H} 2 \mathrm{~B}$, with a preference for modified forms of $\mathrm{H} 2 \mathrm{~A}$ in cells.

Next, we performed in vitro deubiquitylation assays to determine whether USP51 removes ubiquitin from H2Aub. Recombinant mononucleosomes were ubiquitylated by recombinant RNF168 in vitro (Supplemental
Fig. S4A) and used as substrates in deubiquitylation assays. Recombinant USP51, but not USP51/CI, efficiently removed ubiquitin in a time-dependent manner (Fig. 5C). Under the same conditions, USP51 had no apparent activities on H2AK118,119ub isolated from cells stably expressing Flag-H2AK13,15R (Fig. 5D). USP51 could deubiquitylate H2AK13ub (Supplemental Fig. S5A) as well as diubiquitin linked through either Lys 27 or Lys63 of ubiquitin (Supplemental Fig. S5B). Taken together, these results show that USP51 is a DUB for H2AK13,15ub and exhibits enzymatic activities against H2AK15ub from mononucleosomes and ubiquitin chains in vitro.

\section{USP51 regulates the resolution of DNA damage foci}

To investigate the USP51 action in DDR, we first analyzed chromatin binding of USP51 following IR using a chromatin fractionation assay. Chromatin-bound $\gamma$ H2AX increased immediately following IR and then decreased after $1 \mathrm{~h}$, suggesting that some IR-induced breaks are repaired after $1 \mathrm{~h}$. During this time interval, chromatin-bound USP51 was reduced immediately following IR and started to accumulate $1 \mathrm{~h}$ after IR (Fig. 6A). Interestingly, chromatin-bound H2AK15ub was also detected immediately following the reduction of chromatin-bound USP5 1 and was barely detectable $4 \mathrm{~h}$ after IR. Monitoring the formation of IR-induced H2AK15ub foci revealed that depletion of USP51 accelerated the formation of $\mathrm{H} 2 \mathrm{AK} 15 \mathrm{ub}$ foci at early time points and peaked at $4 \mathrm{~h}$ after IR (Supplemental Fig. S6A,B). After 4 h, USP51 depletion resulted in persistent IR-induced H2AK15ub foci compared with control cells (Fig. 6B,C; Supplemental Fig. $\mathrm{S} 6 \mathrm{C})$. These results are consistent with the idea that USP51 is a negative regulator for the formation of IR-induced H2AK15ub foci and also functions to remove H2AK15ub after DNA repair.

RNF168-mediated H2AK15ub is required for the recruitment of downstream proteins, including 53BP1, to DNA damage foci. We next monitored the assembly and disassembly of 53BP1 foci in USP51-depleted U2OS cells as well as USP51 knockout mouse ES cells after IR (Fig. 6D-G; Supplemental Fig. S7). In U2OS cells, USP51 depletion appeared to increase the rate of IR-induced RNF168 and 53BP1 foci formation compared with nontargeting control cells (Supplemental Fig. S7A) during the first hour after IR. Afterward, 53BP1 foci disappeared at a slower rate in both USP51-depleted U2OS cells and USP51 knockout ES cells compared with the corresponding controls (Fig. 6D-G; Supplemental Fig. S7B,C). These results support the idea that USP51 negatively regulates the assembly of DNA damage foci initially and is then required for the removal of H2AK15ub and subsequent disassembly of proteins at DNA damage sites after damaged DNAs are repaired.

\section{USP51 depletion impairs cell viability and repair efficiency}

Next, we asked whether the alterations of USP51 affect cell survival and DNA repair efficiency. USP51-depleted 
Wang et al.
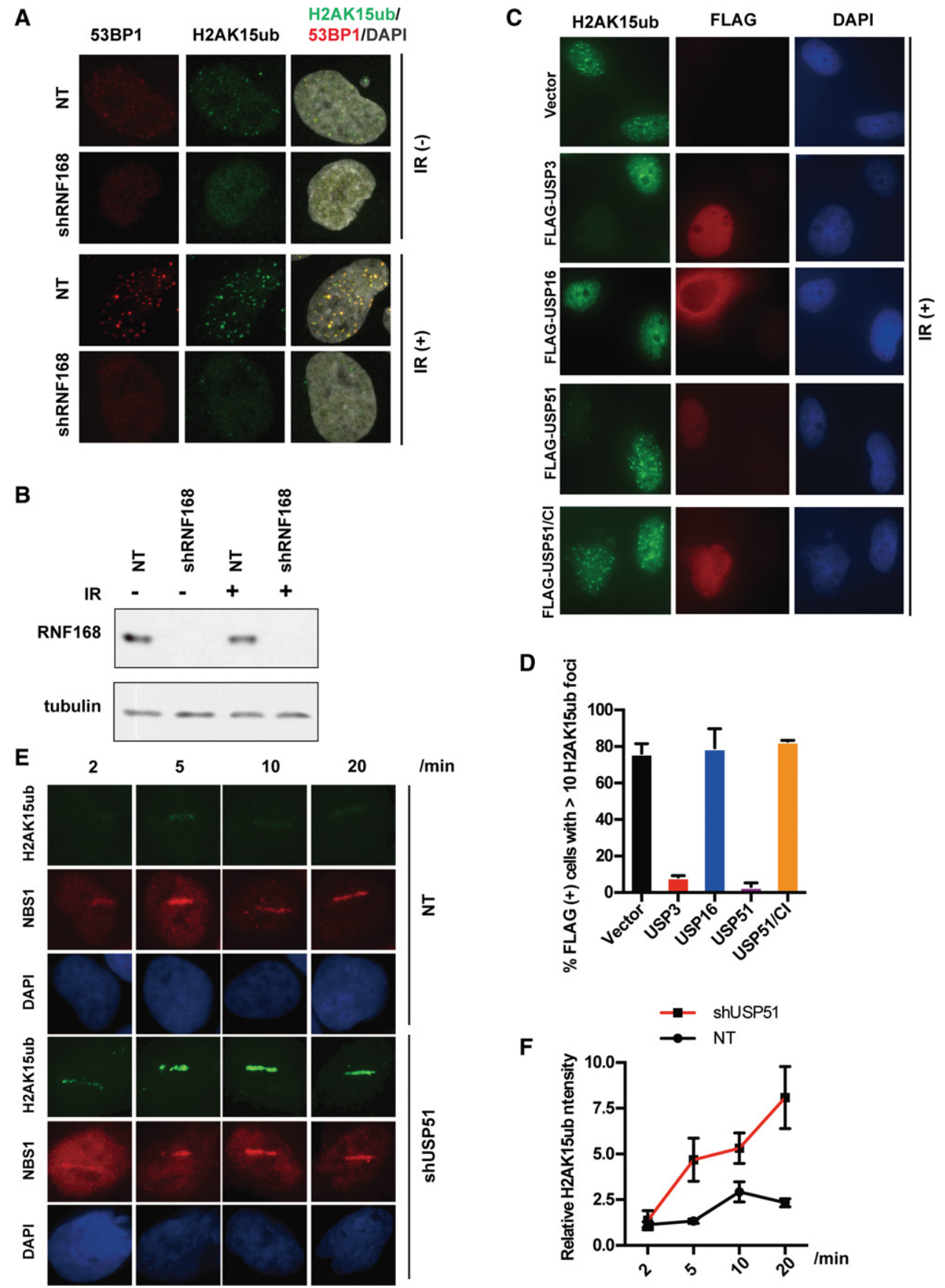

Figure 4. USP51 regulates H2AK15ub levels at DNA damage sites. $(A, B)$ IR-induced H2AK15ub foci colocalize with 53BP1 foci and are dependent on RNF168. U2OS cells with or without RNF168 depletion (shRNF168) were irradiated at 2.5 Gy. After 1 h, immunofluorescence was performed using antibodies against H2AK15ub and 53BP1. Representative images $(A)$ and knockdown efficiency $(B)$ of RNF168 are shown. $(C, D)$ Overexpression of USP3 and USP51 suppresses IR-induced H2AK15ub foci. U2OS cells expressing Flag-USP3, FlagUSP16, or Flag-USP51 were irradiated at $10 \mathrm{~Gy}$. Immunofluorescence images $(C)$ as well as percentage $(D)$ of cells with H2AK15ub foci from three independent experiments are shown. About 50-70 cells from each experiment were counted. $(E, F)$ USP51 depletion results in the accumulation of $\mathrm{H} 2 \mathrm{AK} 15 \mathrm{ub}$ at DNA damage sites induced by laser irradiation. Laser-induced DNA damage strips were marked by immunofluorescence using antibodies against the DSB repair complex protein NBS1. Representative images $(E)$ and relative fluorescence intensity $($ mean $\pm \mathrm{SD})(F)$ from three independent experiments are shown.

U2OS cells and mouse USP51 knockout ES cells exhibited increased sensitivity toward IR (Fig. 7A-C). Expression of human USP51, but not its catalytic inactive mutant in mouse USP51 knockout ES cells, rescued IR sensitivity. Moreover, the increased IR sensitivity in USP51-depleted cells was likely due to increased DNA damage after IR 
A

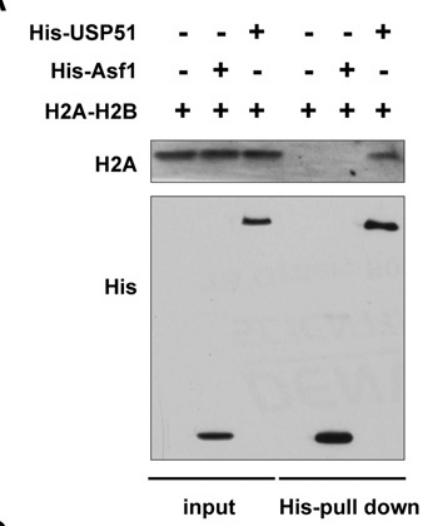

C

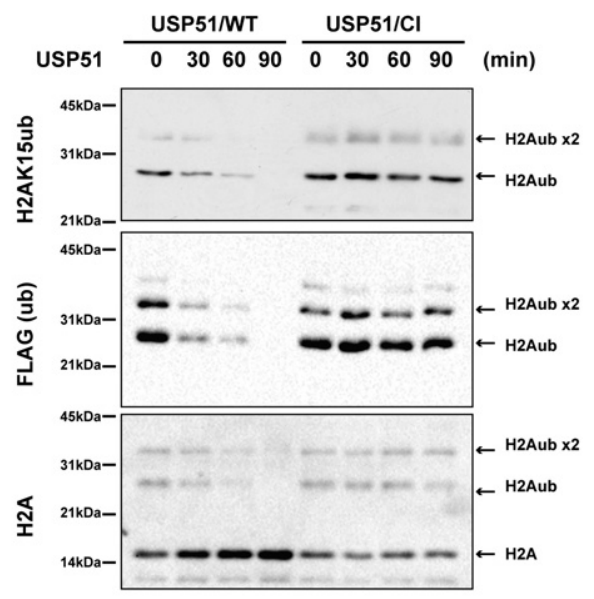

B

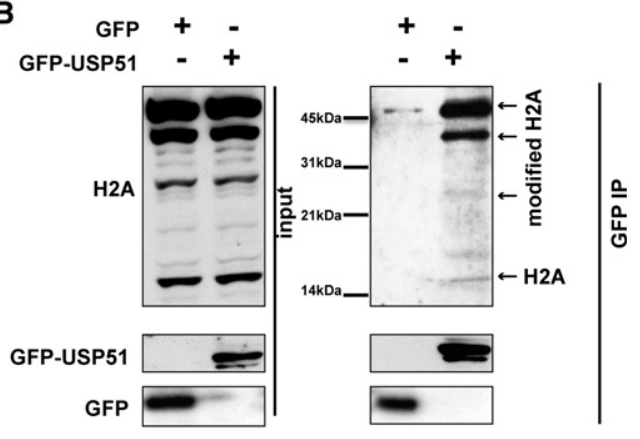

D
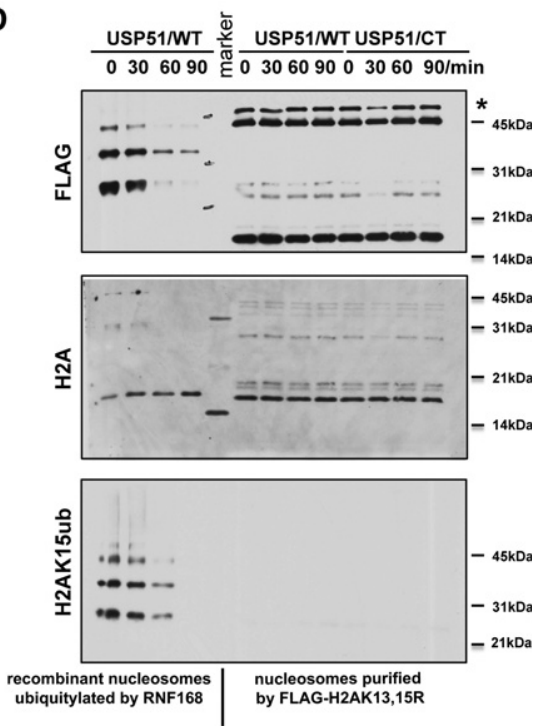

Figure 5. USP51 binds to H2A and deubiquitylates H2AK13,15ub. (A) USP51 binds to H2A in vitro. Purified His-USP51 or His-Asf1 was used to pull down H2A-H2B dimers. Immunoprecipitated proteins were detected by Western blot. (B) USP51 binds to H2A in vivo. GFPUSP51 was transiently transfected to $293 \mathrm{~T}$ cells and immunoprecipitated. Immunoprecipitated proteins were detected by Western blotting using antibodies against H2A. (C) USP51 catalyzes the removal of H2AK13,15ub in vitro. Recombinant mononucleosomes ubiquitylated by RNF168 with Flag-ubiquitin were used as substrates for deubiquitylation reactions with recombinant USP51 or catalytically inactive USP51/CI. The reactions were stopped at the indicated time points with SDS loading buffer, and ubiquitylated species were detected using antibodies against H2A, Flag epitope, and H2AK15ub. (D) USP51 exhibits no enzymatic activity against H2AK118,119ub. Mononucleosomes were immunoprecipitated from 293T cells expressing Flag-H2A K13,15R mutant proteins and used as substrates for deubiquitylation assays. Recombinant mononucleosomes ubiquitylated by RNF168 were used as positive controls. ${ }^{*} \mid$ Nonspecific bonds. Full images for H2AK15ub Western blots in $C$ and $D$ are in Supplemental Figure S10.

(Supplemental Fig. S8A). Interestingly, overexpression of USP51 also led to increased IR sensitivity (Supplemental Fig. S8B). These results indicate that USP51 levels must be regulated in cells to efficiently deal with DNA lesions.

Next, we determined how depletion of USP51 affected NHEJ and HR, two distinct DSB repair pathways to repair IR-induced DNA damage in eukaryotic cells (Bunting et al. 2010), using NHEJ and HR reporter assays (Seluanov et al. 2004; Mao et al. 2007; Patel et al. 2011). Depletion of USP51 resulted in increased NHEJ and HR efficiency compared with controls (Fig. 7D,E). The increased NHEJ and HR efficiencies were likely due to increased H2AK15ub in USP51-depleted cells because they could be antagonized by simultaneous depletion of RNF168 (Supplemental Fig. S9A,B). These results support the idea that USP51 is required for regulating proper DNA repair, most likely through H2AK15ub.

\section{Discussion}

The RNF8-RNF168 ubiquitylation pathway is involved in the recruitment of proteins involved in signaling and DNA repair to the chromatin surrounding DNA damage sites. Here we provide several lines of evidence supporting the model that USP51, a previously uncharacterized DUB, functions downstream from RNF168 E3 ligase and deubiquitylates H2AK15ub after damaged DNA is repaired to restore chromatin to its ground state (Fig. 7F). First, we show that while overexpression of USP51 has no apparent 
Wang et al.

A

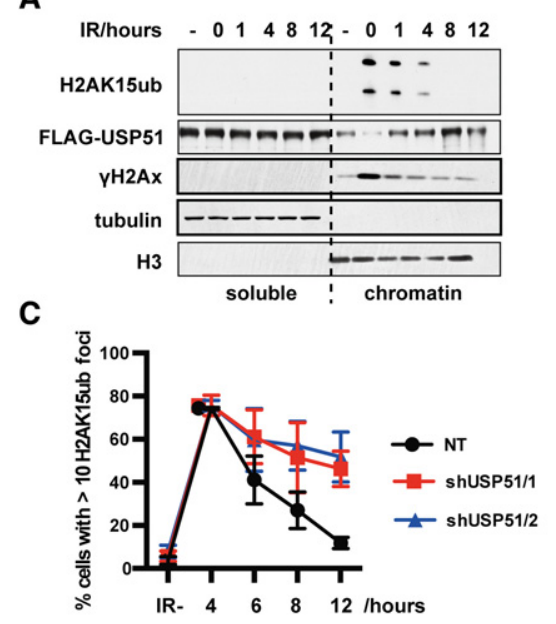

D

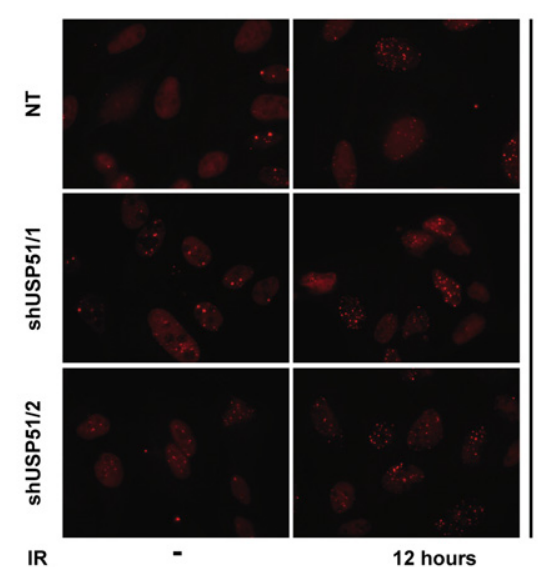

E

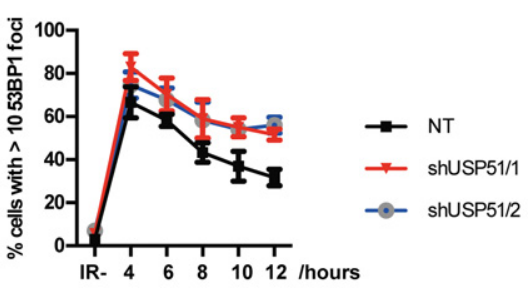

B

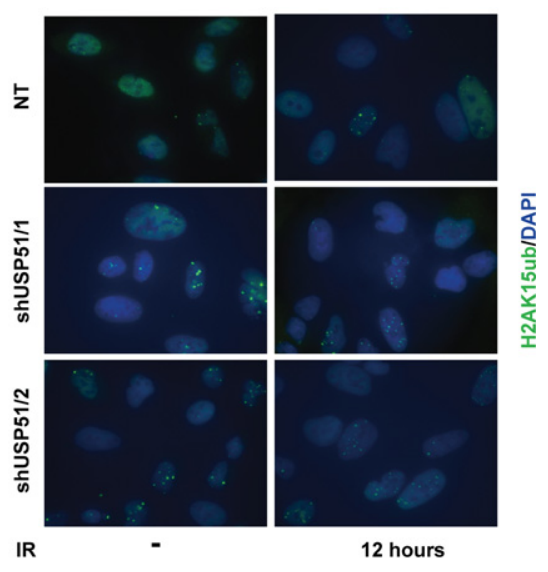

F

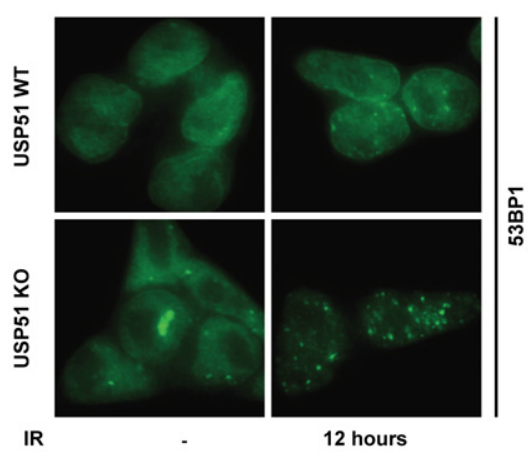

G

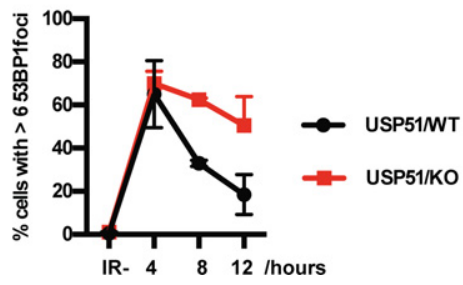

Figure 6. USP51 regulates the resolution of DNA damage foci. (A) Chromatin association of USP51 and H2AK15ub after IR treatment. Chromatin fractionation assays were performed using U2OS cells stably expressing Flag-USP51 with or without 10 Gy of IR. Soluble and chromatin fractions were analyzed by Western blot using the indicated antibodies. A full image of H2AK15ub is in Supplemental Figure S10. $(B, C)$ USP51 depletion results in persistent H2AK15ub foci after IR. USP51-depleted and control U2OS cells were irradiated with 2.5 Gy. Immunofluorescence was performed at the indicated time points after IR treatment using H2AK15ub antibody. (B) Representative immunofluorescence images without IR or $12 \mathrm{~h}$ after IR are shown. (C) The percentage of cells with $>10 \mathrm{H} 2 \mathrm{AK} 15 \mathrm{ub}$ foci at different time points after IR was calculated from three independent experiments. Images acquired at other time points are in Supplemental Figure S6C. $(D, E)$ USP51 depletion results in persistent 53BP1 foci after IR. The experiments were performed as described above. Images acquired at other time points are in Supplemental Figure S7B. $(F, G)$ IR-induced 53BP1 foci were also persistent in USP51 knockout (KO) mouse ES cells. Images acquired at other time points are in Supplemental Figure S7C. About 100 cells in each experiment were counted in $C$ and $E$, and $\sim 50-70$ cells were counted in $G$.

effects on the formation of IR-induced $\gamma \mathrm{H} 2 \mathrm{AX}, \mathrm{MDC1}$, and RNF168 foci, it suppresses the formation of IR-induced 53BP1, BRCA1, and H2AK15ub foci. These results are consistent with a model in which USP51 functions downstream from RNF168 to regulate the levels of H2AK15ub, which is important for the recruitment of
53BP1 and BRCA1 to DNA damage foci. Second, we show that depletion of USP51 results in a dramatic increase in H2AK15ub, whereas overexpression of USP51 leads to a reduction of IR-induced H2AK15ub. Third, USP51 interacts with H2A and removes H2AK15ub but not H2AK119ub in vitro. Fourth, upon DNA damage, 
A

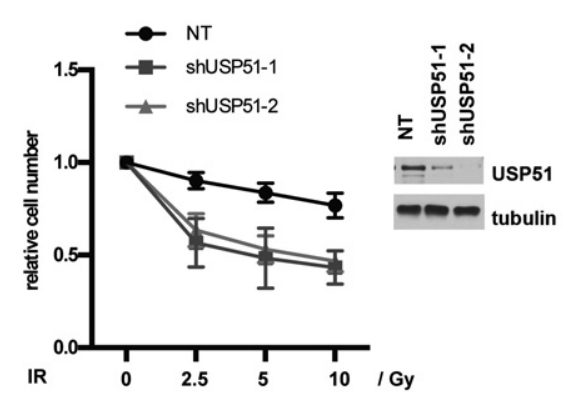

B

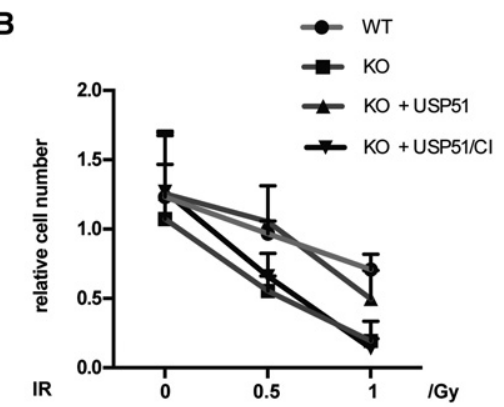

C

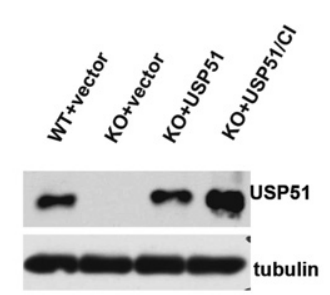

D

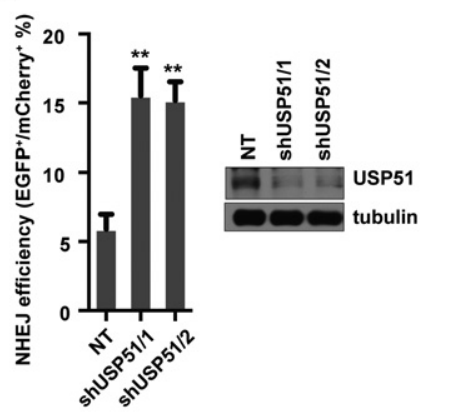

E

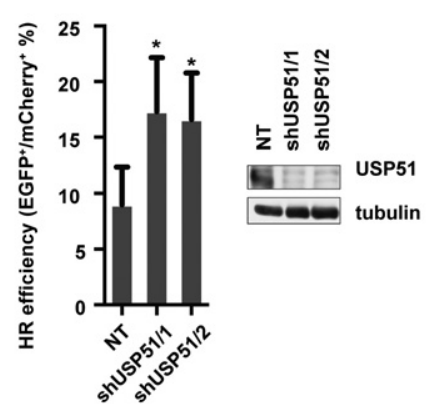

$\mathbf{F}$

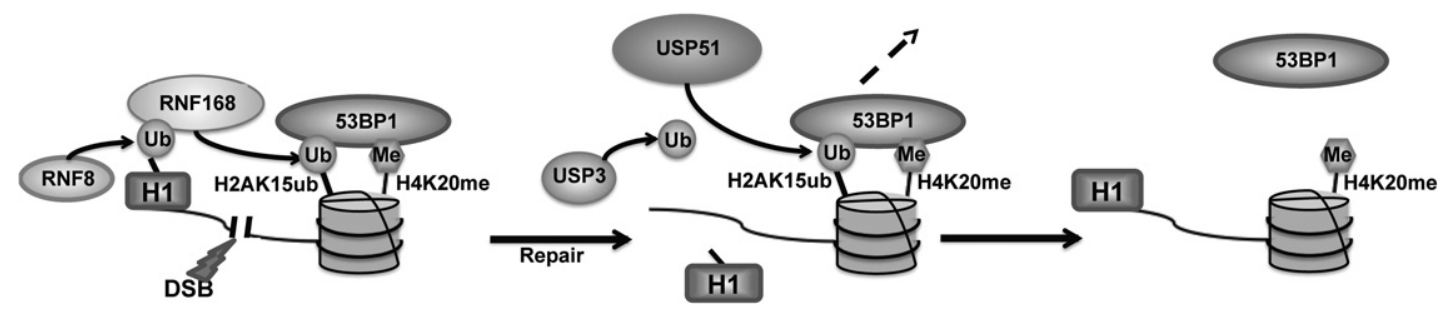

Figure 7. USP51-depleted cells exhibit defects in DNA repair. (A) Depletion of USP51 results in increased sensitivity to IR. U2OS cells infected with USP51 shRNA were irradiated with the indicated IR doses. Cell viability was measured by CellTiter-Blue cell viability assay $3 \mathrm{~d}$ after irradiation. The right panel shows the knockdown efficiency of USP51. $(B, C)$ USP51 knockout mouse ES cells show increased sensitivity to IR compared with wild-type (WT) cells. USP51 knockout (KO) mouse ES cells were infected with USP51, or its catalytically inactive mutants were irradiated with the indicated IR doses. Cell viability was measured $3 \mathrm{~d}$ after irradiation. Exogenously expressed USP51 level is shown in $C$. $(D, E)$ USP51 depletion results in increased NHEJ $(D)$ and HR $(E)$ efficiency. HeLa cells with or without USP51 depletion were used to perform NHEJ assays, and DR-GFP cells were used for HR reporter assays. $(F)$ A model for the role of USP51 in DDR. In response to DSBs, RNF8-RNF168-mediated ubiquitylation cascade leads to H2AK15ub. H2AK15ub facilitates recruitment of 53BP1 and BRCA1 (not shown) to DSBs for DNA repair. After the DSB is repaired, USP51 removes H2AK15ub and facilitates disassembly of 53BP1 at the DSB.

chromatin-bound USP51 disassociates from chromatin immediately following DNA damage and reassociates with chromatin following DNA repair. Together, these results support the idea that USP51 removes H2AK15ub, thereby modulating DDR following repair of DNA damage (Fig. 7F).

It has been shown that the formation of IR-induced RNF168 foci depends on the catalytic activity of RNF168 (Panier et al. 2012). Our result that USP51 overexpression reduces $\mathrm{H} 2 \mathrm{AK} 15 \mathrm{ub}$ but has little effect on the formation of RNF168 foci appears to contradict this observation. One possible explanation is that USP51 overexpression may not completely remove H2AK15ub at IRinduced DNA damage sites, and the residual H2AK15ub along with histone $\mathrm{H} 1$ ubiquitylation catalyzed by RNF8 (Thorslund et al. 2015) can still promote the formation of RNF168 foci.

It has been suggested previously that USP3 and USP16 may target H2AK15ub. Several lines of evidence indicate that the major functions of these two DUBs likely impact other ubiquitylation events. For instance, overexpression of USP3 results in loss of IR-induced RNF168 foci, suggesting that USP3 functions upstream of the recruitment of RNF168 during DDR and that USP3 regulates the ubiquitylation events catalyzed by RNF8. While overexpression of USP3 suppresses IR-induced H2AK15ub levels, the suppression is most likely due to defects in the formation of IR-induced RNF168 foci. Furthermore, overexpression of 
USP16, the major DUB for H2AK119ub, does not affect the formation of IR-induced 53BP1 foci. This observation is consistent with results from one report (Mosbech et al. 2013) but is in contrast to findings from another study (Zhang et al. 2014). The discrepancy of the effect of USP16 overexpression on the formation of IR-induced 53BP foci is likely due to different expression levels in different studies. In the present study, we monitored expression levels of USP3, USP16, and USP51 and found that, when expressed at similar levels, these DUBs had a distinct effect on the formation of RNF168 and 53BP1 foci. In addition, overexpression of UPS16 had no apparent effect on H2AK15ub. These results indicate that while it is possible that USP3 and USP16 regulate the levels of H2AK15ub, USP51 is likely the major DUB targeting H2AK13,15ub, an important histone H2A ubiquitylation associated with DDR.

It has been shown previously that RNF8-RNF168mediated ubiquitylation cascade is tightly regulated during DDR (Jackson and Durocher 2013). We observed for the first time that $\mathrm{H} 2 \mathrm{AK} 15 \mathrm{ub}$ levels increase at DNA damage foci in response to IR, and this increase occurs with a kinetics similar to that of $\gamma-\mathrm{H} 2 \mathrm{AX}$. Interestingly, $\mathrm{H} 2 \mathrm{AK} 15 \mathrm{ub}$ is removed from chromatin after DNA repair.

It is not unprecedented that a histone modification is dynamically controlled during DDR. For instance, in budding yeast, histone H3 Lys56 acetylation (H3K56ac) marks newly synthesized H3. The H3K56ac level peaks during $S$ phase and is deacetylated soon after new H3-H4 is assembled into nucleosomes. It is proposed that the removal of $\mathrm{H} 3 \mathrm{~K} 56 \mathrm{ac}$ is required for the restoration of chromatin after DNA replication. H3K56ac also regulates nucleosome assembly during DNA repair (Chen et al. 2008; Li et al. 2008). It has been shown that the removal of this modification after DNA repair helps turn off checkpoint kinases. Therefore, we suggest that the increase in H2AK15ub immediately after damage helps assemble proteins at DNA damage sites, and its removal after DNA repair not only helps the disassembly of proteins at DNA damage sites but also facilitates the restoration of damaged chromatin to its original state.

\section{UPS51 likely has multiple functions in DDR and/or genome maintenance}

We showed that depletion of USP51 results in increased NHEJ and HR efficiency, which is dependent on the presence of RNF168 in cells. These results suggest that the effect of USP51 depletion on NHEJ and HR is likely due to elevated levels of H2AK15ub at DNA damage sites. The increased NHEJ and HR efficiency in USP51-depleted cells is reminiscent of the effects of depletion of TRIP12 and UBR5, two E3 ligases that negatively regulate RNF168 (Gudjonsson et al. 2012): In cells depleted with TRIP12 and UBR5, RNF168 levels increase, resulting in the spreading of 53BP1 from chromosome break sites and increased NHEJ efficiency (Gudjonsson et al. 2012), possibly due to an increase in H2AK15ub levels. While we did not detect a major expansion of IR-induced 53BP1 foci in USP51-depleted cells, depletion of USP51 re- sults in a delayed disassembly of IR-induced H2AK15ub and 53BP1 foci, which may underlie the observed increases in NHEJ and HR repair efficiency.

One would predict that the increase in NHEJ and HR after USP51 depletion would render cells resistant to IR. In contrast, USP51-depleted cells were sensitive to IR compared with control cells. There are at least two nonmutual explanations for the discordant phenotypes observed for the USP51-depleted cells. First, in addition to removal H2AK15ub after DNA repair, USP51 may function at multiple steps of DDR. Supporting this idea, USP51 disassociates from chromatin immediately following DNA damages and then reassociates with chromatin after DNA repair. Moreover, depletion of USP51 facilitates the formation of H2AK15ub and 53BP1 foci during the early stages of DDR. These results suggest that USP51, in addition to its role in removing H2AK13,15ub after DNA repair, is a negative regulator of initial DDR. Second, in addition to H2AK15ub, USP51 may have other substrates. It is known that most of E3 ubiquitin ligases have more than one substrate (Schwartz and Ciechanover 1999). In principle, a DUB likely has more targets than one E3 ligase because there are far fewer genes encoding DUBs than E3 ligases in the human genome. Therefore, it is quite possible that the cell cycle defects and formation of increased spontaneous DNA foci observed in USP51depleted cells are due to the deregulation of protein ubiquitylation other than H2AK15ub. Future studies are needed to determine these issues and understand how USP51 chromatin disassociation/association is regulated in response to DNA damage.

In summary, we identified USP51, a previously uncharacterized DUB, as a bona fide DUB that removes H2AK13,15ub. Our results indicate that removal of H2AK13,15ub is involved in disassembly of proteins at DNA damage foci after DNA repair and is important for proper DDR and maintenance of genome integrity.

\section{Materials and Methods}

Antibodies

The following antibodies were used in this study: rabbit anti-53BP1 (Rappold et al. 2001), anti- $\gamma \mathrm{H} 2 \mathrm{Ax}$ (Millipore, clone JBW301), rabbit anti- $\gamma-\mathrm{H} 2 \mathrm{AX}$ (Cell Signaling Technologies, 2577), anti-MDC1 (Millipore, clone P2B11), anti-BRCA1 (Santa Cruz Biotechnology, D-9), anti-ubiquitin (Enzo, FK2), anti-Flag (Sigma, M2), rabbit anti-Flag (Sigma, F7425), rabbit anti-EGFP (Abcam, ab6556), anti-tubulin (Developmental Studies Hybridoma Bank, 12G10), and anti-H2A (Millipore, 07-146). Polyclonal anti-USP51 antibody was generated by immunizing the USP51 peptide (144 PRAWRGSRRRSRPG 157) in rabbits and purifying antisera through the USP51 peptide-conjugated beads. Monoclonal anti-H2AK15ub antibody (clone EDL H2AK15-4, IgG $\mathrm{I}_{2 \mathrm{~b}}, \mathrm{k}$ ) was generated in the Mayo Clinic Antibody Hybridoma Core (Supplemental Material).

\section{Mononucleosome immunoprecipitation}

Mononucleosomes were purified as previously described (Chan et al. 2013). Chromatin from the stable cell lines expressing 
Flag-H2A or Flag-H2A mutants were digested by $1 \mathrm{U}$ of micrococcal nuclease per 1000 cells (New England Biolabs, M0247S) for $20 \mathrm{~min}$ at $37^{\circ} \mathrm{C}$. After clarification by centrifugation, the digested nucleosomes were incubated with $30 \mu \mathrm{L}$ of anti-Flag M2 agarose beads (Sigma) overnight at $4{ }^{\circ} \mathrm{C}$. The beads were then washed four times using washing buffer (50 mM HEPES-KOH at $\mathrm{pH} 7.4,200$ $\mathrm{mM} \mathrm{NaCl}, 0.5 \%$ Triton X-100, 10\% glycerol, 100 mM EDTA, proteinase inhibitors) for $5 \mathrm{~min}$ each time. Proteins were eluted by $1 \mathrm{mg} / \mathrm{mL}$ Flag peptide (Sigma) for $30 \mathrm{~min}$ at $16^{\circ} \mathrm{C}$ and precipitated by TCA.

\section{In vitro ubiquitylation and deubiquitylation assays}

In vitro ubiquitylation assays were performed as described previously (Mattiroli et al. 2012; Fradet-Turcotte et al. 2013; Liu et al. 2013). Briefly, $2.5 \mu \mathrm{g}$ of mononucleosomes was incubated with $30 \mathrm{nM}$ yeast E1, $1.5 \mu \mathrm{M}$ UbcH5a, $4 \mu \mathrm{M}$ RNF168 (1-113), $10 \mu \mathrm{M}$ Flag-ubiquitin, and $3 \mathrm{mM}$ ATP in ubiquitylation buffer $(50 \mathrm{mM}$ Tris- $\mathrm{HCl}$ at $\mathrm{pH} 7.5,100 \mathrm{mM} \mathrm{NaCl}, 10 \mathrm{mM} \mathrm{MgCl} 2,1 \mathrm{mM} \mathrm{ZnCl}_{2}$, $1 \mathrm{mM} \mathrm{DTT}$ ) for $2 \mathrm{~h}$ at $30^{\circ} \mathrm{C}$. The ubiquitylated nucleosomes were then diluted with deubiquitylation buffer and immunoprecipitated with anti-Flag M2 beads. The immunoprecipitated mononucleosomes were eluted with Flag peptide and used as substrates in the deubiquitylation assays and the screen of monoclonal antibody against H2AK15ub. The ubiquitylated nucleosomes were incubated with $10 \mathrm{ng} / \mu \mathrm{L}$ purified USP51 or USP51/CI for the indicated time points at $30^{\circ} \mathrm{C}$. After deubiquitylation reactions, proteins were precipitated by TCA, resuspended in $1 \times$ SDS loading buffer, resolved on SDS-PAGE, and detected by Western blot using the Flag antibody.

\section{Assays for NHEJ and HR efficiency}

NHEJ was performed as described previously (Patel et al. 2011). Briefly, HeLa cells were infected with the lentivirus for USP51 shRNA or nontarget shRNA control. After selection for $2 \mathrm{~d}$, cells were cotransfected with pmCherry and linearized Pem1-EGFPAd2. pmCherry was used to normalize transfection efficiency. Cells were collected for FACS analysis, and GFP-positive cells were counted to determine the NHEJ efficiency. The ratio between $\mathrm{GFP}^{+}$and mCherry ${ }^{+}$cells was used as a measure of NHEJ efficiency. HR assays were performed following published procedures (Luo et al. 2012). Briefly, HeLa-DR-GFP cells were infected with the lentivirus for USP51 shRNA or nontarget shRNA control. After $2 \mathrm{~d}$ of selection, cells were cotransfected with I-SceI (pCBA-I-SceI) endonuclease to induce DSB and pmCherry to control transfection efficiency. Cells were harvested $2 \mathrm{~d}$ after I-SceI transfection and subjected to flow cytometric analysis. The ratio between $\mathrm{GFP}^{+}$and $\mathrm{mCherry}^{+}$cells was used as a measure of DSB repair efficiency.

\section{Laser irradiation and immunofluorescence staining}

U2OS cells were cultured on 35-mm glass-bottomed dishes (MatTek Cultureware, P35G-15-14-C). Cells were then infected with lentivirus expressing shUSP51 or nontarget control. Forty-eight hours after infection, DNA damage was introduced to cells via irradiation with 551-nm dye laser in a line pattern. A customized laser microirradiation system was previously described (Pei et al. 2011; Chen et al. 2013). Briefly, the system consists of an inverted microscope (Nikon), a laser ablation unit (Photonic Instruments), and microscope automation and imaging software (Metamorph, Molecular Devices). An attenuator plate (50\% transmission) and four pulses per spot were used to set the total laser energy delivered to each focused spot in the line pat- tern. Immediately following laser irradiation, cells were fixed with $4 \%$ paraformaldehyde (Electron Microscopy Sciences) for $10 \mathrm{~min}$ and then permeabilized with phosphate-buffered saline (PBS) containing $0.2 \%$ Triton X-100 for $10 \mathrm{~min}$ at room temperature. Immunofluorescence staining was then performed as described previously (You et al. 2009). Mouse antibodies against H2AK15ub were used at 1:100, and rabbit antibodies against NBS1 were used at 1:200. Primary antibodies were detected with goat anti-mouse Alexa fluor 488-conjugated and goat antirabbit Alexa fluor 568-conjugated secondary antibodies. DNA was visualized using $1 \mu \mathrm{g} / \mathrm{mL}$ Hoechst 33342 staining. Cells were imaged using the Nikon microscope and the MetaMorph software described above. The relative signal intensity of $\mathrm{H} 2 \mathrm{AK} 15 \mathrm{ub}$ on the laser-induced DNA damage strips was quantified as the H2AK15ub intensity normalized to that of NBS1 (control). Signal intensity of H2AK15ub or NBS1 was measured as the fold increases relative to the background signal in an adjacent region outside the strip but within the nucleus. For each laser strip signal, an average of three random positions on the strip was compared with an average of three random positions on the background, and then relative signals were determined for each time point and experimental condition. Quantified results shown in Figure $4 \mathrm{~F}$ were obtained from three independent experiments. Error bars in the figures indicate standard deviation.

\section{Acknowledgments}

We thank Dr. Xiaochun Yu for plasmids expressing RNF168 and RNF168 antibody. We thank Dr. Daniel Durocher for plasmids expressing RNF169 and antibodies. These studies are supported by National Institutes of Health grants (GM81838 and CA157489 to Z.Z., and GM098535 to Z.Y.) and an American Cancer Society Research Scholar grant (RSG-13-212-01-DMC).

\section{References}

Botuyan MV, Lee J, Ward IM, Kim JE, Thompson JR, Chen J, Mer G. 2006. Structural basis for the methylation state-specific recognition of histone $\mathrm{H} 4-\mathrm{K} 20$ by 53BP1 and Crb2 in DNA repair. Cell 127: 1361-1373.

Branzei D, Foiani M. 2008. Regulation of DNA repair throughout the cell cycle. Nat Rev Mol Cell Biol 9: 297-308.

Bunting SF, Callen E, Wong N, Chen HT, Polato F, Gunn A, Bothmer A, Feldhahn N, Fernandez-Capetillo O, Cao L, et al. 2010. 53BP1 inhibits homologous recombination in Brca1-deficient cells by blocking resection of DNA breaks. Cell 141: 243-254.

Chan KM, Fang D, Gan H, Hashizume R, Yu C, Schroeder M, Gupta N, Mueller S, James CD, Jenkins R, et al. 2013. The histone $\mathrm{H} 3.3 \mathrm{~K} 27 \mathrm{M}$ mutation in pediatric glioma reprograms H3K27 methylation and gene expression. Genes Dev 27: 985-990.

Chen CC, Carson JJ, Feser J, Tamburini B, Zabaronick S, Linger J, Tyler JK. 2008. Acetylated lysine 56 on histone H3 drives chromatin assembly after repair and signals for the completion of repair. Cell 134: 231-243.

Chen X, Paudyal SC, Chin RI, You Z. 2013. PCNA promotes processive DNA end resection by Exo1. Nucleic Acids Res 41: 9325-9338.

Ciccia A, Elledge SJ. 2010. The DNA damage response: making it safe to play with knives. Mol Cell 40: 179-204.

Doil C, Mailand N, Bekker-Jensen S, Menard P, Larsen DH, Pepperkok R, Ellenberg J, Panier S, Durocher D, Bartek J, et al. 2009. RNF168 binds and amplifies ubiquitin conjugates on 
damaged chromosomes to allow accumulation of repair proteins. Cell 136: 435-446.

Fradet-Turcotte A, Canny MD, Escribano-Diaz C, Orthwein A, Leung CC, Huang H, Landry MC, Kitevski-LeBlanc J, Noordermeer SM, Sicheri F, et al. 2013. 53BP1 is a reader of the DNA-damage-induced H2A Lys 15 ubiquitin mark. Nature 499: $50-54$.

Gatti M, Pinato S, Maspero E, Soffientini P, Polo S, Penengo L. 2012. A novel ubiquitin mark at the $\mathrm{N}$-terminal tail of histone H2As targeted by RNF168 ubiquitin ligase. Cell Cycle 11: $2538-2544$.

Ghosal G, Chen J. 2013. DNA damage tolerance: a doubleedged sword guarding the genome. Transl Cancer Res 2: 107-129.

Gudjonsson T, Altmeyer M, Savic V, Toledo L, Dinant C, Grofte M, Bartkova J, Poulsen M, Oka Y, Bekker-Jensen S, et al. 2012. TRIP12 and UBR5 suppress spreading of chromatin ubiquitylation at damaged chromosomes. Cell 150: 697-709.

Huen MS, Chen J. 2010. Assembly of checkpoint and repair machineries at DNA damage sites. Trends Biochem Sci 35: 101-108.

Huen MS, Grant R, Manke I, Minn K, Yu X, Yaffe MB, Chen J. 2007. RNF8 transduces the DNA-damage signal via histone ubiquitylation and checkpoint protein assembly. Cell 131: 901-914.

Jackson SP, Bartek J. 2009. The DNA-damage response in human biology and disease. Nature 461: 1071-1078.

Jackson SP, Durocher D. 2013. Regulation of DNA damage responses by ubiquitin and SUMO. Mol Cell 49: 795-807.

Joo HY, Zhai L, Yang C, Nie S, Erdjument-Bromage H, Tempst P, Chang C, Wang H. 2007. Regulation of cell cycle progression and gene expression by H2A deubiquitination. Nature 449: $1068-1072$.

Kolas NK, Chapman JR, Nakada S, Ylanko J, Chahwan R, Sweeney FD, Panier S, Mendez M, Wildenhain J, Thomson TM, et al. 2007. Orchestration of the DNA-damage response by the RNF8 ubiquitin ligase. Science 318: 1637-1640.

Lancini C, van den Berk PC, Vissers JH, Gargiulo G, Song JY, Hulsman D, Serresi M, Tanger E, Blom M, Vens C, et al. 2014. Tight regulation of ubiquitin-mediated DNA damage response by USP3 preserves the functional integrity of hematopoietic stem cells. J Exp Med 211: 1759-1777.

Li Q, Zhou H, Wurtele H, Davies B, Horazdovsky B, Verreault A, Zhang Z. 2008. Acetylation of histone H3 lysine 56 regulates replication-coupled nucleosome assembly. Cell 134: 244-255.

Liu C, Wang D, Wu J, Keller J, Ma T, Yu X. 2013. RNF168 forms a functional complex with RAD6 during the DNA damage response. J Cell Sci 126: 2042-2051.

Luo K, Zhang H, Wang L, Yuan J, Lou Z. 2012. Sumoylation of $\mathrm{MDC1}$ is important for proper DNA damage response. EMBO I 31: 3008-3019.

Mailand N, Bekker-Jensen S, Faustrup H, Melander F, Bartek J, Lukas C, Lukas J. 2007. RNF8 ubiquitylates histones at DNA double-strand breaks and promotes assembly of repair proteins. Cell 131: 887-900.

Mao Z, Seluanov A, Jiang Y, Gorbunova V. 2007. TRF2 is required for repair of nontelomeric DNA double-strand breaks by homologous recombination. Proc Natl Acad Sci 104: 1306813073.

Marechal A, Zou L. 2013. DNA damage sensing by the ATM and ATR kinases. Cold Spring Harb Perspect Biol 5: a012716.

Mattiroli F, Vissers JH, van Dijk WJ, Ikpa P, Citterio E, Vermeulen W, Marteijn JA, Sixma TK. 2012. RNF168 ubiquitinates K13-
15 on $\mathrm{H} 2 \mathrm{~A} / \mathrm{H} 2 \mathrm{AX}$ to drive DNA damage signaling. Cell 150: $1182-1195$.

Mosbech A, Lukas C, Bekker-Jensen S, Mailand N. 2013. The deubiquitylating enzyme USP44 counteracts the DNA doublestrand break response mediated by the RNF8 and RNF168 ubiquitin ligases. I Biol Chem 288: 16579-16587.

Nakada S, Tai I, Panier S, Al-Hakim A, Iemura S, Juang YC, O'Donnell L, Kumakubo A, Munro M, Sicheri F, et al. 2010. Non-canonical inhibition of DNA damage-dependent ubiquitination by OTUB1. Nature 466: 941-946.

Nicassio F, Corrado N, Vissers JH, Areces LB, Bergink S, Marteijn JA, Geverts B, Houtsmuller AB, Vermeulen W, Di Fiore PP, et al. 2007. Human USP3 is a chromatin modifier required for S phase progression and genome stability. Curr Biol 17: 1972-1977.

Panier S, Boulton SJ. 2014. Double-strand break repair: 53BP1 comes into focus. Nat Rev Mol Cell Biol 15: 7-18.

Panier S, Ichijima Y, Fradet-Turcotte A, Leung CC, Kaustov L, Arrowsmith CH, Durocher D. 2012. Tandem protein interaction modules organize the ubiquitin-dependent response to DNA double-strand breaks. Mol Cell 47: 383-395.

Patel AG, Sarkaria JN, Kaufmann SH. 2011. Nonhomologous end joining drives poly(ADP-ribose) polymerase (PARP) inhibitor lethality in homologous recombination-deficient cells. Proc Natl Acad Sci 108: 3406-3411.

Pei H, Zhang L, Luo K, Qin Y, Chesi M, Fei F, Bergsagel PL, Wang L, You Z, Lou Z. 2011. MMSET regulates histone H4K20 methylation and 53BP1 accumulation at DNA damage sites. Nature 470: 124-128.

Poulsen M, Lukas C, Lukas J, Bekker-Jensen S, Mailand N. 2012. Human RNF169 is a negative regulator of the ubiquitin-dependent response to DNA double-strand breaks. I Cell Biol 197: 189-199.

Rappold I, Iwabuchi K, Date T, Chen J. 2001. Tumor suppressor p53 binding protein 1 (53BP1) is involved in DNA damage-signaling pathways. J Cell Biol 153: 613-620.

Schwartz AL, Ciechanover A. 1999. The ubiquitin-proteasome pathway and pathogenesis of human diseases. Annu Rev Med 50: 57-74.

Seluanov A, Mittelman D, Pereira-Smith OM, Wilson JH, Gorbunova V. 2004. DNA end joining becomes less efficient and more error-prone during cellular senescence. Proc Natl Acad Sci 101: 7624-7629.

Shanbhag NM, Rafalska-Metcalf IU, Balane-Bolivar C, Janicki SM, Greenberg RA. 2010. ATM-dependent chromatin changes silence transcription in cis to DNA double-strand breaks. Cell 141: 970-981.

Shao G, Lilli DR, Patterson-Fortin J, Coleman KA, Morrissey DE, Greenberg RA. 2009. The Rap80-BRCC36 de-ubiquitinating enzyme complex antagonizes RNF8-Ubc13-dependent ubiquitination events at DNA double strand breaks. Proc Natl Acad Sci 106: 3166-3171.

Sharma N, Zhu Q, Wani G, He J, Wang QE, Wani AA. 2014. USP3 counteracts RNF168 via deubiquitinating $\mathrm{H} 2 \mathrm{~A}$ and $\gamma \mathrm{H} 2 \mathrm{AX}$ at lysine 13 and 15. Cell Cycle 13: 106-114.

Stewart GS, Panier S, Townsend K, Al-Hakim AK, Kolas NK, Miller ES, Nakada S, Ylanko J, Olivarius S, Mendez M, et al. 2009. The RIDDLE syndrome protein mediates a ubiquitin-dependent signaling cascade at sites of DNA damage. Cell 136: 420-434.

Thorslund T, Ripplinger A, Hoffmann S, Wild T, Uckelmann M, Villumsen B, Narita T, Sixma TK, Choudhary C, Bekker-Jensen S, et al. 2015. Histone H1 couples initiation and amplification of ubiquitin signalling after DNA damage. Nature 527: 389-393. 
Wang B, Elledge SJ. 2007. Ubc13/Rnf8 ubiquitin ligases control foci formation of the Rap80/Abraxas/Brca1/Brcc36 complex in response to DNA damage. Proc Natl Acad Sci 104: 20759-20763.

You Z, Shi LZ, Zhu Q, Wu P, Zhang YW, Basilio A, Tonnu N, Verma IM, Berns MW, Hunter T. 2009. CtIP links DNA double-strand break sensing to resection. Mol Cell 36: 954-969.

Zhang Z, Yang H, Wang H. 2014. The histone H2A deubiquitinase USP16 interacts with HERC2 and fine-tunes cellular response to DNA damage. I Biol Chem 289: 3288332894 . 


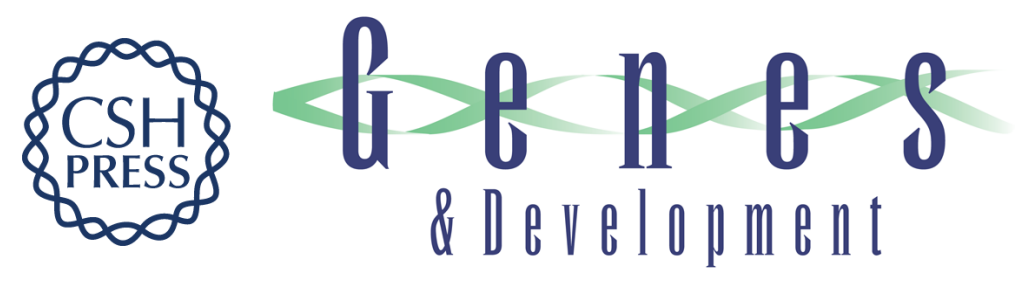

\title{
USP51 deubiquitylates H2AK13,15ub and regulates DNA damage response
}

\author{
Zhiquan Wang, Honglian Zhang, Ji Liu, et al.
}

Genes Dev. 2016, 30:

Access the most recent version at doi:10.1101/gad.271841.115

\section{Supplemental http://genesdev.cshlp.org/content/suppl/2016/04/15/30.8.946.DC1 Material}

References This article cites 45 articles, 14 of which can be accessed free at: http://genesdev.cshlp.org/content/30/8/946.full.html\#ref-list-1

Creative This article is distributed exclusively by Cold Spring Harbor Laboratory Press for the first Commons six months after the full-issue publication date (see

License http://genesdev.cshlp.org/site/misc/terms.xhtml). After six months, it is available under a Creative Commons License (Attribution-NonCommercial 4.0 International), as described at http://creativecommons.org/licenses/by-nc/4.0/.

Email Alerting Receive free email alerts when new articles cite this article - sign up in the box at the top Service right corner of the article or click here.

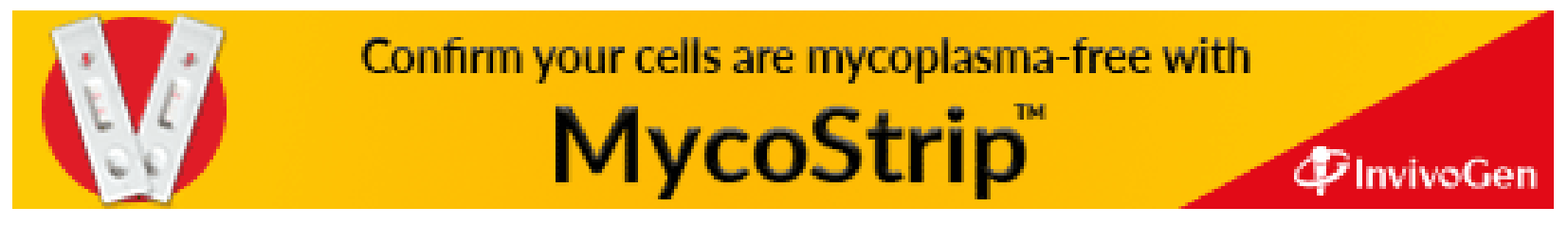

